\title{
Eorlier the Better: Interceptive Orthodontics to Correct Vorious forms of Malocclusion in a Nine-year-old Boy
}

\author{
Dr Amita Rai,' Dr Bandana Koirala,2 Dr Mamta Dali,3 Dr Sneha Shrestha4 \\ 'Consultant Pediatric Dentist, Dental Department, Kanti Children's Hospital, Maharajgunj, Kathmandu, Nepal \\ 2Professor, Head, ${ }^{3}$ Associate Professor, ${ }^{4}$ Assistant professor, \\ Department of Pedodontics \& Preventive Dentistry, College of Dental Surgery, B.P. Koirala Institute of Health Sciences, Dharan, Nepal.
}

Correspondence: Dr Amita Rai; Email: amitarai2013@gmail.com

\section{ABSTRACT}

Guidance of eruption and development of the primary, mixed, and permanent dentitions is an integral component of comprehensive oral healthcare for all pediatric dental patients; which should contribute to the development of a permanent dentition that is stable, and functionally and esthetically acceptable. In this case note, we present a case in which various forms of interceptive treatment modalities like; extraction of impacted and erupted supernumerary teeth, correction of anterior crossbite and irregularities using Z-springs and Hawley's labial bow, and provision of lingual holding arch space maintainer were done to correct malocclusion in a nine-year-old patient. Arch alignment was achieved within seven month of starting of treatment, which was acceptable to the patient.

Keywords: Crossbite, Interceptive orthodontics, Lingual holding arch, Supernumerary teeth

\section{INTRODUCTION}

Guidance of eruption and development of the primary, mixed, and permanent dentitions is an integral component of comprehensive oral healthcare for all pediatric dental patients. Such guidance should contribute to the development of a permanent dentition that is stable, and functionally and esthetically acceptable.' Here, we present a case in which various forms of interceptive treatment modalities like; extraction of impacted and erupted supernumerary teeth (ST), correction of anterior crossbite (AC) and irregularities using Z-springs and Hawley's labial bow, and provision of lingual holding arch (LHA) space maintainer (SM) were done to correct malocclusion in a nine-year-old patient.

\section{CASE REPORT}

A nine-year-old male patient reported with chief complaint of irregular upper front teeth. Intraoral examination revealed: crossbite wrt. 1221 (Figure 1A); deep pit and fissure on: 55653646 (Figure 1B, 1C); grossly carious 74758485 (Figure 1C); ST palatal to 11 (Figure 1B); lingually tilted 32 (Figure 1A); and mobile: $\begin{array}{llllll}73 & 74 & 75 & 83 & 84 & 85\end{array}$. Occlusal radiograph revealed additional inverted ST palatal to 2122 (Figure 1D).
Informed and written consent from the guardian, and assent from the patient was taken prior to starting treatment. After medical consultations and complete hematological investigations, comprehensive dental treatment was provided to the patient which included: oral hygiene instructions; diet counseling; topical fluoride application; pit and fissure sealant on 556536 46; oral prophylaxis; extraction of ST (Figure 2) 737475 838485 under local anesthesia; Hawley's retainer to correct the proclined 11 along with Z-springs on 12 and 21 with posterior bite plane (Figure 3E, 3F); and LHA SM (Figure 3G).

The patient was recalled every three weeks for activation of Z-springs. Crossbite correction and proper incisor alignment was achieved within two months. On seven month follow-up, eruption of mandibular permanent canines and premolars were noted, and proper lower incisor alignment was achieved (Figure $4 \mathrm{H}, 4 \mathrm{I})$. In this visit, insertion of new LHA was done on this visit to accommodate for the lower incisor alignment. Again, LHA with U-loop was inserted on 18-month follow-up visit for the proper alignment of slightly rotated 33 (Figure $4 \mathrm{~K}$ ). 

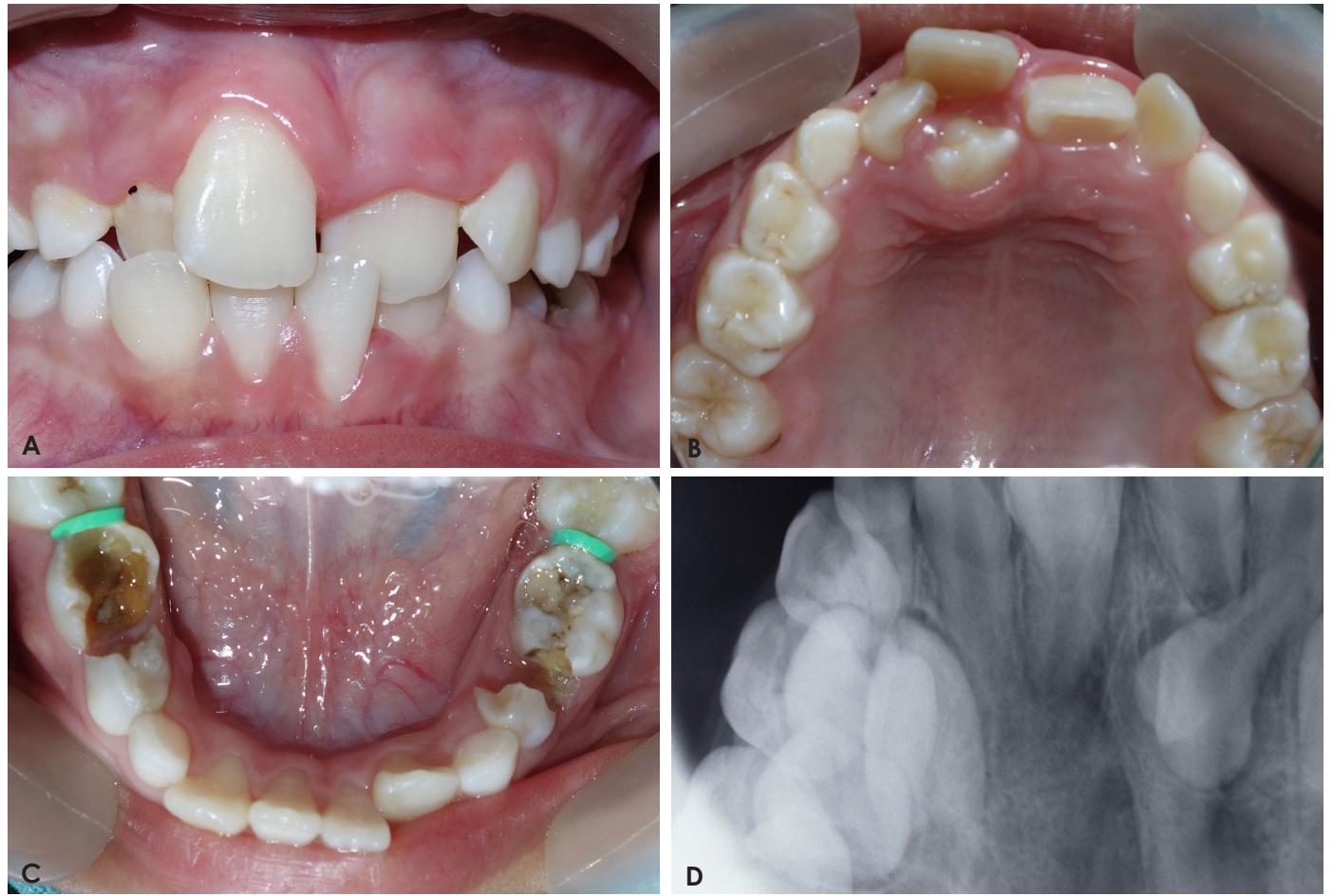

Figure 1: Pre-treatment records
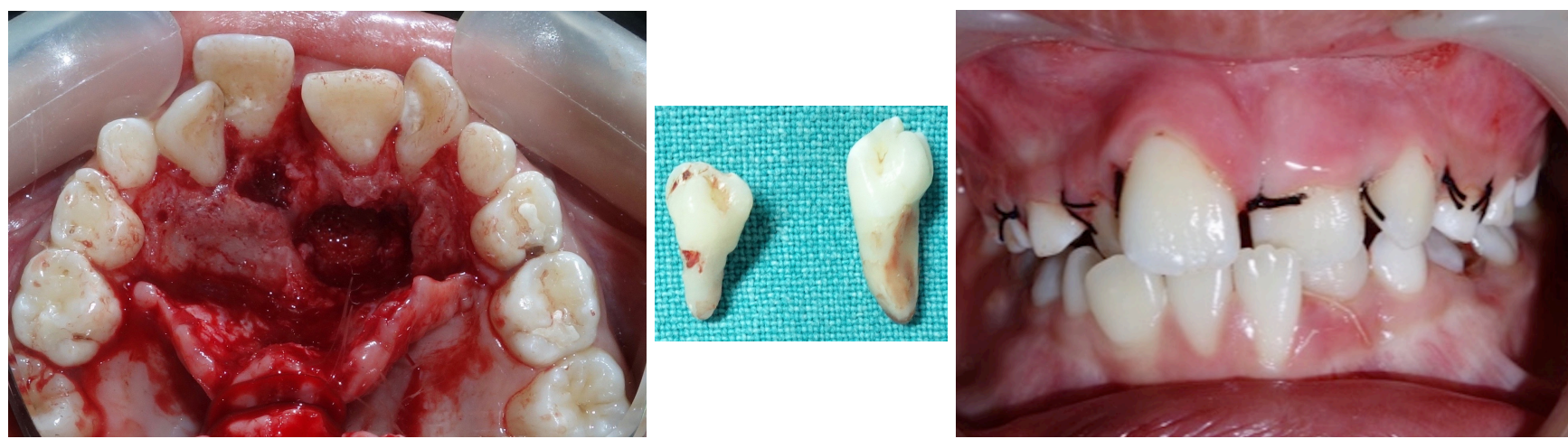

Figure 2: Surgical Removal of Supernumerary teeth
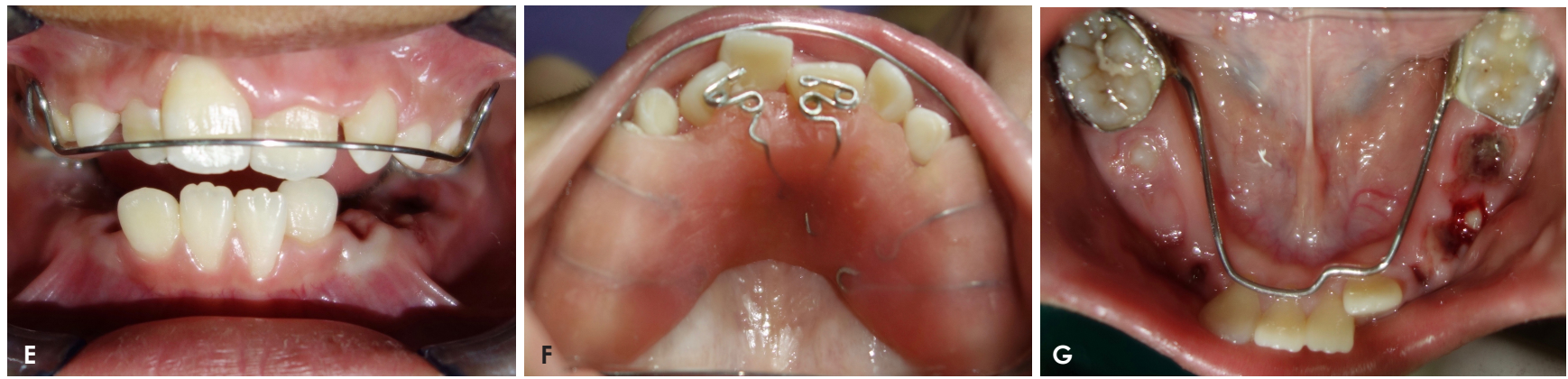

Figure 3: Appliance Therapy 

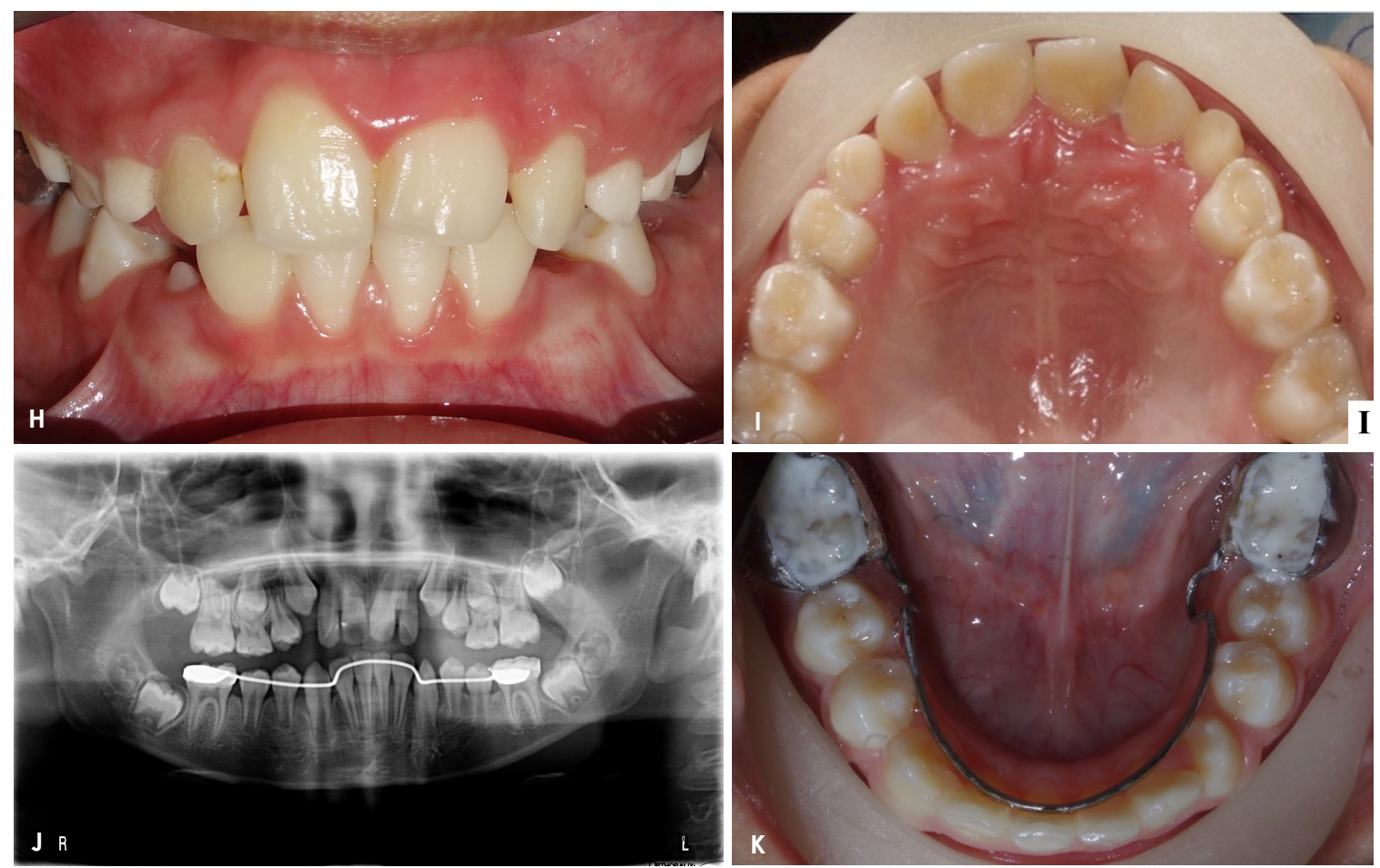

Figure 4: Post Treatment records

\section{DISCUSSION}

American Academy of Orthodontics in 1969 defined interceptive orthodontics (IO) as, "that phase of the science and art of orthodontics employed to recognize and eliminate potential irregularities and malpositions in the developing dentofacial complex". Children who benefit from 10 has been reported to be $14-49 \%$. Various malocclusion which would be benefited from IO include: crossbite, supernumerary teeth, oral habits, crowding, scissor bite, space loss, etc. ${ }^{2}$

Preventive and interceptive orthodontic treatment facilitates the population's access to treatment, allowing the elimination of some etiologic factors and preventing the progression of dental, skeletal and functional disharmonies, mitigating or even eliminating the need for more complex treatments thereafter. ${ }^{3,4}$ A thorough clinical examination, appropriate pretreatment records, differential diagnosis, sequential treatment plan, and progress records are necessary to manage any condition affecting the developing dentition.'

Patient factors such as immaturity, lack of motivation or parental supervision, small mouth size, low pain threshold and poor oral hygiene could influence the success of the 1O. Interceptive orthodontic treatment often requires follow-up treatment in the permanent dentition. ${ }^{4}$ The goals and objectives of 10 must be established firmly in order to prevent unnecessary, prolonged treatment that may burn out the patient in the second phase treatment later. ${ }^{2}$

An anterior crossbite is defined as, "an abnormal reversed labiolingual relationship of the incisors, where one or more primary or permanent maxillary incisors are located palatally to the mandibular incisors" ${ }^{5.6}$ Anterior crossbite can be classified as: dental, functional and skeletal crossbite. ${ }^{5}$ The prevalence of the AC varies between $2.2 \%$ and $12 \% .5 .6$ Anterior croccbite can lead to an adverse complication including gingival recession and mobility, TMJ disorders, as well as dental and facial disharmony. Therefore, it's highly recommended to correct an $A C$ in the deciduous or early mixed dentition to allow a normal development of the occlusion and jaws. ${ }^{5}$ Once identified, the treatment should ideally involve a technique which is simple, non-invasive, involves little chairside time, requires minimal patient cooperation and gives rapid correction of the crossbite. In young patients, compliance can be an issue, but appearance less so. Therefore, the types of intervention need to be different than those used on an adolescent patient, where aesthetics and appearance usually provide the 
treatment motivation. ${ }^{\circ}$ If enough space is available a simple AC can be aligned as soon as the condition is noted. Treatment options include acrylic incline planes, acrylic retainers with lingual springs, bonded resin-composite slopes, fixed appliances with springs, rare earth magnetic appliances, multiple sets of Essixbased appliances. If space is needed, an expansion appliance also is an option. ${ }^{7.8}$

Removable appliances have the advantages of easier maintenance and oral hygiene care for young patients, utilization of palatal anchorage, and the ability to move a selected block of teeth. ${ }^{8}$ The disadvantage of removable appliance is the requirement of patient compliance and longer duration of treatment. ${ }^{5}$ In our case, we had used Hawley's retainer to correct the proclined 11 along with Z-springs on 12 and 21 with posterior bite plane. The posterior bite planes allows for disocclusion of the anterior teeth in crossbite to allow them to move freely over the bite following a force application by either a spring or by tongue pressure. ${ }^{5}$

Supernumerary teeth is the terms to describe an excess in tooth number. They are thought to be related to disturbances in the initiation and proliferation stages of dental development.' Supernumerary teeth may vary from a simple odontoma, through a conical or tuberculate tooth, to a supplemental tooth which closely resembles a normal tooth. ${ }^{9}$ The prevalence of ST has been found to be $0.2 \%-0.8 \%$ and $0.5 \%-5.3 \%$ in deciduous and permanent dentition, respectively. The male-to-female ratio for the incidence was reported to range in between $1.18: 1$ and $1.5: 1.10$ Supernumerary teeth are more frequently observed in permanent dentition than in deciduous dentition with more frequency for the upper arch than lower arch in a proportion of 10:1.1,11 The diagnosis of a ST can be confirmed with radiographs, including occlusal, periapical, or panoramic films, or computed tomography.

Management of ST teeth depends on the type and position of the tooth. 9 Controversy exists regarding the optimal treatment time and treatment modality for impacted ST. Immediate removal of mesiodens is usually indicated in the situations like; inhibition or delay of eruption, displacement of the adjacent tooth, interference with orthodontic appliances, presence of pathologic condition, or spontaneous eruption of the ST. ${ }^{2}$ In our case, the impacted and erupted ST were interfering with the proper arch alignment in the premaxillary area. Timely removal of ST facilitated for the proper function of orthodontic appliance, resulting in desired arch alignment in our case.

The most common orthodontic problem encountered today is the lack of space in the permanent dentition. Early loss of deciduous teeth causes shifting of adjacent teeth into the space created resulting in abnormal axial inclination, spacing between teeth and shifting of dental midline. This prevents the normal eruption of their permanent successors from their normal eruption pathways leading to malocclusion. The term space maintenance was coined by JC Braver in 1941. He defined space maintenance as the, "process of maintaining a space in a dental arch previously occupied by a tooth or a group of teeth". The objectives of SM are preservation of primate space, integrity of the dental arches, normal occlusal planes, and to aid in esthetics and phonetics. A successfully restored carious tooth is the best SM. ${ }^{13}$ Whenever possible, restoration of carious primary teeth should be attempted to avoid malocclusions that could result from their extraction. American Academy of Pediatric Dentistry (AAPD) recommends the use of SM to reduce the prevalence and severity of malocclusion following premature loss of primary teeth.

There are various types of SMs, among which LHA is a bilateral, fixed, non-functional SM used in mandibular arch. The LHA arch also has design variations. In addition to the laboratory fabricated custom design, these appliances also come in premade forms like; premade wires designed to fit in horizontal tubes welded to the lingual surfaces of the molar bands and lingual arch wires designed to fit into vertical tubes. ${ }^{14}$ In this case, we opted for custom designed LHA which was cemented on 36 46. Provision of LHA preserved the spaces created after the extraction of mobile canines and non-restorable molars. The SMs need regular follow-up and sometime they require fabrication of new SM also. In our case, we changed LHA three times, to accommodate for the arch alignment, and to regain space for the rotated 73 .

The LHA can be used in situations where there are multiple missing teeth. Because it does not contact any primary teeth, it is less likely to interfere with the increase in inter-canine width. By resisting mesial movement of the permanent first molars, the LHA arch 
also has the capacity to relieve potential crowding by allowing incisors to drift distally into the leeway space. There are some disadvantages of LHA like interference with the eruption of the permanent incisors if placed before the loss of the primary incisors, proclination of the lower incisors, preservation of leeway space which may increase the prevalence of permanent second molar impactions. ${ }^{14}$

The charm of 10 is that timely diagnosis and treatment of malocclusion helps to avoid complex orthodontic treatment in the future. In addition, it helps build-up confidence in a growing child who has just started to become aware of his/her appearance. In our case, the parents of the child expressed that the treatment outcome had positive impact in behavior and confidence of the patient. Every treatment we provide, outcome depends upon the treating doctor as well as the patient compliance. In our case, patient compliance in terms of wearing appliance, following the instructions, and visiting from distant village for regular follow-up was very motivating to us as well.

\section{REFERENCES}

1. American Academy of Pediatric Dentistry Guideline on Pediatric Oral Surgery. 2010;32(6):238-45.

2. Srinivas N. Interceptive orthodontics-a short review. Research \& Reviews: A Journal of Dentistry. 2011;2(1): 6-9.

3. Siqueira MA, ZotarFilho IJ, NaimKassis E, Da Rosa APB, de Araujo TSB, Ramires MA, Buchala CACN, Preventive and Interceptive Orthodontics: Review of its Importance. International Journal of Development Research. 2015;5(10):5784-8.

4. King GJ and Brudvik P. Effectiveness of Interceptive Orthodontic Treatment in Reducing Malocclusions. American Journal of Orthodontics and Dentofacial Orthopedics. 2010;137(1):18-25.

5. Khalaf K, Mando M. Removable Appliances to Correct Anterior Crossbites in the Mixed Dentition: a Systematic Review. Acta Odontologica Scandinavica. 2019;1-8.

6. Borrie F, Bearn D. Early Correction of Anterior Crossbites: A Systematic Review. Journal of Orthodontics. 201 1;38:175-84.

7. American Academy of Pediatric Dentistry Guideline on Management of the Developing Dentition and Occlusion in Pediatric Dentistry. 2014;38(6):289-301

8. Bindayel NA. Simple Removable Appliances to Correct Anterior and Posterior Crossbite in Mixed Dentition: Case Report. The Saudi Dental Journal. 2012;24:105-13.

9. Meighani G, Pakdaman A. Diagnosis and Management of Supernumerary (Mesiodens): A Review of the Literature. Journal of Dentistry, Tehran University of Medical Sciences. 2010;7(1):41-9.

10. Mahto RK, Dixit S, Kafle D, Agarwal A, Bornstein M, Dulal S. Nonsyndromic Bilateral Posterior Maxillary Supernumerary Teeth: A Report of Two Cases and Review. Case Reports in Dentistry. 2018:1-6.

11. Acharya S, Ghosh C, Mondal PK. Bilateral Supernumerary Teeth in Deciduous Dentition-A Rarity. Journal of Clinical and Diagnostic Research. 2014:8(5):18-9.

12. Mallineni SK, Nuvvula S. Management of supernumerary teeth in children: A narrative overview of published Literature. Journal of CranioMaxillary Diseases. 2015;4(1):62-8.

13. Singh PH, Naorem H, Devi TC, Debbarma N. Modern Concepts of Space Maintainers and Space Regainers: A Review Article. European Journal of Pharmaceutical and Medical Research. 2020;7(3).

14. Jitesh S, Mathew MG. Space Maintainer- A Review. Drug Invention Today. 2019;1 1(1):21-5. 